\title{
RNA interference targeting Aurora-A sensitizes glioblastoma cells to temozolomide chemotherapy
}

\author{
JING GAN $^{1,2}$, FANGFANG WANG $^{1,2}$, DEZHI MU $^{1,2}$, YI QU $^{1,2}$, RONG LUO $^{1}$ and QIU WANG $^{1}$ \\ ${ }^{1}$ Department of Pediatrics, West China Second University Hospital; \\ ${ }^{2}$ Key Laboratory of Obstetric \& Gynecologic and Pediatric Diseases and Birth Defects of Ministry of Education, \\ Sichuan University, Chengdu, Sichuan 610041, P.R. China
}

Received July 3, 2015; Accepted August 25, 2016

DOI: 10.3892/ol.2016.5261

\begin{abstract}
Clinically, temozolomide (TMZ) is widely used in glioblastoma (GBM) treatment. However, the toxicity of TMZ may influence the quality of patient life. Thus, novel treatment options for sensitizing GBM cells to TMZ chemotherapy are necessary. Aurora-A is widely expressed in GBM and correlated with poor prognosis. It has been proven to be an effective target for gene therapy and chemotherapy. In the present study, short hairpin (sh)RNA targeting Aurora-A was employed to knockdown Aurora-A expression in GBM cells. Cell Counting Kit- 8 assays, flow cytometry, colony formation assays, invasion assays and tube formation assays were used to determine the effects of Aurora-A knockdown when combined with TMZ treatment. A U251 subcutaneous cancer model was established to evaluate the efficacy of combined therapy. The results of the present study indicated that the proliferation, colony formation, invasion and angiogenesis of GBM cells were significantly inhibited by combined therapy when compared with TMZ treatment alone. In vivo results demonstrated that knockdown of Aurora-A significantly $(\mathrm{P}=0.0084)$ sensitizes GBM cells to TMZ chemotherapy. The results of the present study demonstrated that knockdown of Aurora-A in GBM cells enhances TMZ sensitivity in vitro and in vivo. Therefore, Aurora-A knockdown may be a novel treatment option for decreasing TMZ toxicity and improving patient quality of life.
\end{abstract}

\section{Introduction}

Glioblastoma (GBM) is a devastating form of brain cancer with a poor median survival time, high level of resistance to current therapy and common recurrence following treatment (1). The

Correspondence to: Dr Qiu Wang or Dr Rong Luo, Department of Pediatrics, West China Second University Hospital, Sichuan University, 20, Section 3, Renmin Nanlu, Chengdu, Sichuan 610041, P.R. China

E-mail: 1109329811@qq.com

E-mail: 25099988@qq.com

Key words: temozolomide, Aurora-A, glioblastoma median survival time is 15 months, and this has improved little over the past four decades (2). The current standard therapy for GBM includes maximum debunking surgery, radiation and treatment with the monofunctional alkylating agent temozolomide (TMZ), also referred to as $\operatorname{Temodar}^{\circledR}(3)$. TMZ is a chemotherapeutic DNA-methylating agent useful in the treatment of GBM (4). DNA O6-methylguanine (O6MG) lesions created by drug exposure incorrectly pair with thymine during the first cycle of DNA replication, leading to activation of the DNA mismatch repair system (5). The removal of the thymine opposite O6MG and subsequent reinsertion following repair resynthesis leads to cycles of futile mismatch repair (5). This ultimately leads to DNA single-strand breaks, activation of sensors of DNA damage, the generation of DNA double-strand breaks, and delayed cell death by a combination of senescence and mitotic catastrophe $(6,7)$. Despite its ability to prolong disease-free survival, the toxicity of TMZ treatment at typical doses has been proven to affect patient quality of life $(8,9)$. Thus, novel treatment options for sensitizing GBM cells to TMZ chemotherapy are necessary.

Aurora-A is a serine/threonine kinase critical for centrosome duplication, spindle assembly and mitotic exit (10-12). Human Aurora-A maps to chromosomal region 20q13.2, which is amplified in various types of cancer $(12,13)$. Aurora-A is also overexpressed in terms of mRNA and protein levels in various types of cancer. Ectopic overexpression of Aurora-A has been shown to transform rodent cell lines $(14,15)$, establishing it as a proto-oncogene. Aurora-A has been documented to be involved in p53 regulation as it phosphorylates p53 at Ser315, leading to its mouse double minute 2-mediated ubiquitination and subsequent proteolysis (16). Aurora-A also drives cell cycle progression by promoting cyclin B1, Wnt, myc and other pro-proliferative signaling pathways (17-19). Repression of Aurora-A by RNA interference delays tetraploidy, apoptosis and senescence in several types of cancer cell $(20,21)$.

In the present study, it was hypothesized that knockdown of Aurora-A in GBM cells would greatly enhance their sensitivity to TMZ. Thus, in vitro short hairpin (sh)RNA targeting Aurora-A was demonstrated to be an appropriate tool to knockdown Aurora-A expression at the protein and mRNA level in U251 cells. Subsequently, Cell Counting Kit-8 (CCK8) assays, flow cytometric analysis, colony formation assays, invasion assays and tube formation assays were 
performed and demonstrated that knockdown of Aurora-A sensitizes GBM cells to TMZ in vitro. Furthermore, TMZ treatment combined with knockdown of Aurora-A by plasmid based shAurora-A/liposome complex significantly inhibited U251 subcutaneous tumor growth, compared with TMZ treatment alone. The results of the present study demonstrated that knockdown of Aurora-A in GBM cells enhanced TMZ sensitivity in vitro and in vivo. Aurora-A knockdown may be a novel treatment option in order to decrease TMZ toxicity.

\section{Materials and methods}

Vector construction. Plasmids were constructed through usage of the pGensil-2 parental vector (Genesil Biotechnology Co., Wuhan, China). Oligonucleotide sequences of Aurora-A-shRNA (CACCGATGCCCTGTCTTACTG TCATTCAAGAGATGACAGTAAGACAGGGCATTT

TTTG) were designed by Genesil Biotechnology Co. according to a published sequence of Aurora-A-shRNA, which was shown to efficiently silence Aurora-A expression in vitro and in vivo (22). Oligonucleotide sequences of scramble-shRNA, which had no homology with any of the mammalian sequence (CACCGCGTACGCGGAATACTTCGATTCAAGAGA TCGAAGTATTCCGCGTACGTTTTTG), were designed as a negative control. The resulting recombinant plasmids were named shAurora-A or shControl (shCtrl), respectively. The two constructs were verified by DNA sequencing. Plasmids were extracted using EndoFree Plasmid Giga kits (Qiagen GmbH, Hilden, Germany) from DH5a Escherichia coli transformants and stored at $-20^{\circ} \mathrm{C}$ until use. The concentration was determined by measuring the $\mathrm{A}_{260} / \mathrm{A}_{280}$ ratio using UV spectrophotometry.

Cell line and treatment. The U251 and U87-MG cell lines were obtained from American Type Culture Collection (Manassas, VA, USA). U251 and U87-MG cells were cultured in Dulbecco's Modified Eagle's Medium (DMEM) containing $10 \%$ fetal bovine serum (FBS) (Gibco; Thermo Fisher Scientific, Inc., Waltham, MA, USA). All cells were maintained in a humidified atmosphere containing $5 \% \mathrm{CO}_{2}$ at $37^{\circ} \mathrm{C}$. Cell transfection was performed using FuGENE ${ }^{\circledR}$ HP Transfection Reagent (Roche Diagnostics, Indianapolis, IN, USA) according to the manufacturer's protocol. Briefly, cells were seeded into 6 -well plates at a density of $2 \times 10^{5}$ cells/well and cultured for $24 \mathrm{~h}$ to reach $70-80 \%$ confluency. A total of $2 \mu \mathrm{g}$ plasmid was diluted in $100 \mu \mathrm{l}$ media without serum and $5 \mu 1 \mathrm{FuGENE}^{\circledR} \mathrm{HP}$ Transfection Reagent was added to the tubes containing the diluted DNA. These were subsequently mixed and the transfection complex incubated for $15 \mathrm{~min}$ at room temperature, before being added to the 6 -well plates. Medium alone was used as blank control. Simultaneously, cells were treated with a dose of $10 \mu \mathrm{M}$ TMZ. Cells and cell supernatant were harvested $48 \mathrm{~h}$ subsequent to transfection for reverse transcription-polymerase chain reaction (RT-PCR) analysis, western blotting, colony formation assays, cell proliferation assays and human umbilical vein endothelial cells (HUVEC) tube formation analysis. All treatments were performed in triplicate.

RNA extraction and RT-PCR. Total RNA was extracted from cells using TRIzol ${ }^{\circledR}$ reagent (Invitrogen; Thermo Fisher
Scientific, Inc.). RNA samples ( $1 \mu \mathrm{g})$ were subjected to reverse transcription using the Takara Primescript RT-PCR kit (Takara Bio, Inc., Otsu, Japan). The primer sequences used were as follows: Aurora-A (515 bp) 5'-GAGGCAGTGGGCTTTGG-3' (sense) and 5'-GGCAGGTAGTCCAGGGTG-3' (antisense). RT-PCR was performed with reverse transcription at $50^{\circ} \mathrm{C}$ for $30 \mathrm{~min}$, followed by initial denaturation at $94^{\circ} \mathrm{C}$ for $3 \mathrm{~min}$ and 30 cycles of $30 \mathrm{sec}$ at $94^{\circ} \mathrm{C}, 30 \mathrm{sec}$ at $60^{\circ} \mathrm{C}$ and $45 \mathrm{sec}$ at $72^{\circ} \mathrm{C}$. All PCR products were separated by electrophoresis on $1 \%$ agarose gels and visualized using ethidium bromide. The amplified products were quantified by Quantity One software (Version 4.1; Bio-Rad Laboratories, Inc., Hercules, CA, USA). Experiments were performed in triplicate.

Western blotting. Cells were lysed on ice for 30 min with radioimmunoprecipitation assay lysis buffer [containing $50 \mathrm{mM}$ Tris- $\mathrm{HCl}, \mathrm{pH} 7.4 ; 1 \%$ NP-40, $0.25 \%$ Na-deoxycholate, $150 \mathrm{mM} \mathrm{NaCl}, 1 \mathrm{mM}$ ethylenediaminetetraacetic acid (EDTA), $1 \mathrm{mM}$ phenylmethane sulfonyl fluoride, $1 \mu \mathrm{g} / \mathrm{ml}$ aprotinin, $1 \mu \mathrm{g} / \mathrm{ml}$ leupeptin, $1 \mu \mathrm{g} / \mathrm{ml}$ pepstatin, $1 \mathrm{mM} \mathrm{Na}_{3} \mathrm{VO}_{4}$ and $1 \mathrm{mM} \mathrm{NaF}$. The protein concentration was determined by the bicinchoninic acid assay (Beyotime Institute of Biotechnology, Haimen, China), and the proteins $(25 \mu \mathrm{g})$ were separated by sodium dodecyl sulfate-polyacrylamide gel electrophoresis and electronically transferred onto a polyvinylidene difluoride membrane (EMD Millipore, Billerica, MA, USA). Following blocking in tris-buffered saline and Tween 20 (TBST) buffer containing $5 \%$ milk, the membranes were incubated with primary antibodies against Aurora-A (catalog no., 14475; dilution, 1:500; Cell Signaling Technology, Inc., Danvers, MA, USA), p53 (catalog no., 2524; dilution, 1:1,200; Cell Signaling Technology, Inc.), caspase 3 (catalog no., 9661; dilution, 1:600; Cell Signaling Technology, Inc.), matrix metalloproteinase-2 (MMP-2) (catalog no., 4022; dilution, 1:800; Cell Signaling Technology, Inc.), MMP-9 (catalog no., 2270; dilution, dilution, 1:1,000; Cell Signaling Technology, Inc.) and glyceraldehyde 3-phosphate dehydrogenase (GAPDH) (catalog no., sc-25778; dilution, 1:5,000; Santa Cruz Biotechnology, Inc., Dallas, TX, USA) at $4^{\circ} \mathrm{C}$ overnight, followed by washing with TBST buffer and incubation with the following horseradish peroxidase-conjugated secondary antibodies: Goat anti-mouse immunoglobulin G (catalog no., ab97040; dilution, 1:5,000) or goat anti-rabbit (catalog no., ab191866; dilution, 1:5,000; Abcam, Cambridge, MA, USA) at $37^{\circ} \mathrm{C}$ for $1 \mathrm{~h}$. Bands were visualized using an enhanced chemiluminescence kit (EMD Millipore). The ratio of Aurora-A/GAPDH was calculated by using densitometry (on Image J; Version 1.0, National Institutes of Health, Bethseda, MD, USA) and values were normalized by dividing by the ratio at blank (23).

Flow cytometric analysis (FCM). FCM was performed to identify apoptosis-positive cells by propidium iodide (PI) (Beyotime Institute of Biotechnology) staining. Briefly, cells were harvested with trypsin-EDTA, washed with phosphate buffered saline (PBS) twice, and pelleted (by centrifugation at 1,200 $\mathrm{x} g$ for $3 \mathrm{~min}$ at $4^{\circ} \mathrm{C}$ ) and suspended in $500 \mu \mathrm{l}$ binding buffer. A total of $5 \mu \mathrm{PI}$ was subsequently added. Following incubation at room temperature for $15 \mathrm{~min}$, analysis was performed on a FACSCalibur system (BD Biosciences, San Jose, CA, USA). Sub-G1 cells were classified as apoptosis positive cells. 
Cell proliferation assay. U251 and U87-MG cells were seeded into 96 well plates at a density of $5 \times 10^{3}$ cells per well in $200 \mu \mathrm{l}$ medium. Cell proliferation was monitored using the CCK8 assay (Dojindo Molecular Technologies, Inc., Kumamoto, Japan). Briefly, $10 \mu$ l CCK8 solution was added into each well, and $4 \mathrm{~h}$ later, the absorbance was determined at a wavelength of $450 \mathrm{~nm}$. Experiments were performed in triplicate.

Colony formation assay. Transfected cells were seeded into 6-well plates at a density of $1 \times 10^{3}$ cells per well. After 7 days of incubation at $37^{\circ} \mathrm{C}$ the cells were fixed with $100 \%$ methanol, stained with $0.05 \%$ crystal violet (Beyotime Institute of Biotechnology) and washed with PBS. The colonies were counted with an Olympus BX600 microscope in at least six randomly chosen fields. The results of three independent experiments are given.

In vitro tube formation assay. The wells of a 96-well plate were coated with ice-cold Matrigel $^{\mathrm{TM}}$ (BD Biosciences). Following polymerization of the matrix at $37^{\circ} \mathrm{C}$, HUVECs isolated in the method described by Dai et al (24) were seeded onto the polymerized extracellular matrix at a density of $2 \times 10^{4}$ cells in $100 \mu 1$ of extracellular growth medium-2 (Lonza, Walkersville, MD, USA) per well; $100 \mu 1$ of the cell supernatant collected from the transfected cells was immediately added. The tube branches were photographed using a Nikon Eclipse Ti-U following $6 \mathrm{~h}$ of incubation. The capillary-like structures of the HUVECs were counted in three randomly selected fields. The results of three independent experiments are presented.

Invasion assay. The invasion of U251 cells was evaluated using 24-well Transwells ${ }^{\circledR}$ (8-lm poresize; EMD Millipore), as described previously but with slight modification (25). Briefly, the filter of the Transwell plate was coated with $50 \mu$ l Matrigel. Following Matrigel polymerization, $500 \mu 1$ DMEM containing $10 \%$ FBS was placed in the lower chamber and $100 \mu 1 \mathrm{U} 251$ suspension $\left(2 \times 10^{4}\right.$ cells/well; in DMEM without $10 \%$ FBS $)$ was placed in the upper chamber. Following incubation for $48 \mathrm{~h}$, cells on the lower surface were fixed in $100 \%$ methanol, stained with $0.05 \%$ crystal violet, counted in at least six randomly selected fields and photographed using an Olympus BX600 microscope.

Enzyme-linked immunosorbent assay (ELISA). A total of $48 \mathrm{~h}$ after transfection, cell supernatants were collected and stored at $-80^{\circ} \mathrm{C}$. The supernatant was used to measure the total levels of several cytokines using the human vascular endothelial growth factor (VEGF) ELISA kit (NeoBioscience, Shenzhen, China) according to the manufacturer's protocol.

Cationic liposome and preparation of plasmid DNA/lipoplexes. Cationic liposome was prepared as multilamellar vesicles for in vivo use as described previously (24). Briefly, 1,2 dioleoyl-3-trimethylammonium-propoane (Avanti Polar Lipids, Inc. Alabaster, AL, USA) and cholesterol (Sigma-Aldrich; EMD Millipore) were mixed in a 1:1 molar ratio, dehydrated in round-bottom tubes using a freeze dryer (SCIENTZ-50ND; Scientz Biotechnology Co., Ltd., Ningbo, China), then rehydrated in $5 \%$ glucose solution by heating at $50^{\circ} \mathrm{C}$ for $6 \mathrm{~h}$. For the in vivo injection, plasmid DNA/lipoplexes were prepared immediately prior to injection by gently mixing cationic liposome with plasmid DNA at a ratio of $12.5 \mu \mathrm{g}$ total cationic liposome to $2.5 \mu \mathrm{g}$ plasmid DNA, resulting in a final concentration of $12.5 \mu \mathrm{g}$ plasmid DNA per $\mathrm{ml}$ in a sterile solution of $5 \%$ glucose in water.

Tumor growth and treatments in vivo. To establish a U251 subcutaneous cancer model, nude female BALB/c mice (6-8 weeks old; $18-20 \mathrm{~g}$ weight) were obtained from The Animal Center of Sichuan University (Chengdu, China) and housed in a $26^{\circ} \mathrm{C}$ and $50 \%$ humidity-controlled facility, with a $24 \mathrm{~h}$ light/dark cycle and free access to food and water. A total of $5 \times 10^{6} \mathrm{U} 251$ cells were injected subcutaneously into the right flank regions of nude female BALB/c mice (6-8 weeks old). After one week, when the tumors were palpable, the animals were randomly assigned to five independent groups, each group containing five mice, and subjected to systemic treatment with one of the following: shCtrl, dimethyl sulfoxide (DMSO), shAurora-A, TMZ (Sigma-Aldrich; EMD Millipore) and shAurora-A+TMZ. Plasmid DNA $(2.5 \mu \mathrm{g})$ and cationic liposome $(12.5 \mu \mathrm{g})$, in $100 \mu \mathrm{l}$ of $5 \%$ glucose was injected into the mouse tail vein three times per week (on Mondays, Wednesdays and Fridays). TMZ was intraperitoneally injected once every 3 days at a dose of $200 \mathrm{mg} / \mathrm{kg}$. Tumor diameters were measured once every three days during the treatment period. Tumor volume was estimated using the following formula: Tumor volume $\left(\mathrm{mm}^{3}\right)=\operatorname{length}(\mathrm{mm}) \times[\mathrm{wi}$ $\operatorname{dth}(\mathrm{mm})]^{2} \times 1 / 2$. The weight, appetite and behavior of the mice were observed. Mice were sacrificed through cervical dislocation following 10 series of treatment and dissected tumors were weighed. Animal studies were performed in accordance with the Institutional Animal Care and Treatment Committee of Sichuan University (Chengdu, China), and written ethical approval was also provided by this board.

Immunostaining. Expression of cluster of differentiation 31 (CD31) was measured with a rat anti-mouse CD31 antibody (catalog no., 550674; BD Biosciences). Tumor sections (3-5 $\mu \mathrm{m})$ of frozen tissues were mounted on 3-aminopropyl triethoxysilane-coated glass slides. Sections were fixed with $4 \%$ paraformaldehyde and washed with PBS ( $\mathrm{pH}$ 7.4). Endogenous peroxide was blocked with $3 \% \mathrm{H}_{2} \mathrm{O}_{2}$ for $10 \mathrm{~min}$. Following PBS washes, slides were blocked with $5 \%$ normal goat serum (Beijing Zhongshan Golden Bridge Biotechnology Co., Ltd., Beijing, China) in PBS for 15 min at room temperature, followed by incubation with primary anti-CD31 (1:400) antibody in blocking solution overnight at $4^{\circ} \mathrm{C}$. All slides were subsequently incubated with a 1:200 dilution of biotin-conjugated goat anti-rat secondary antibody (catalog no., PV6004; Beijing Zhongshan Golden Bridge Biotechnology Co., Ltd.) for $15 \mathrm{~min}$ at $37^{\circ} \mathrm{C}$ and streptavidin-biotin complex at $37^{\circ} \mathrm{C}$ for $15 \mathrm{~min}$. The immunoreaction was visualized using diaminobenzidine peroxide solution (Beijing Zhongshan Golden Bridge Biotechnology Co., Ltd.) and cellular nuclei were counterstained with hematoxylin (Beyotime Institute of Biotechnology). All specimens were evaluated using the Olympus BX600 microscope and Spot Fiex camera. Control samples exposed to secondary antibody alone exhibited no specific staining.

TUNEL. To detect apoptotic cells in tumor tissue, the TUNEL assay was performed according to the manufacturer's protocol 
A
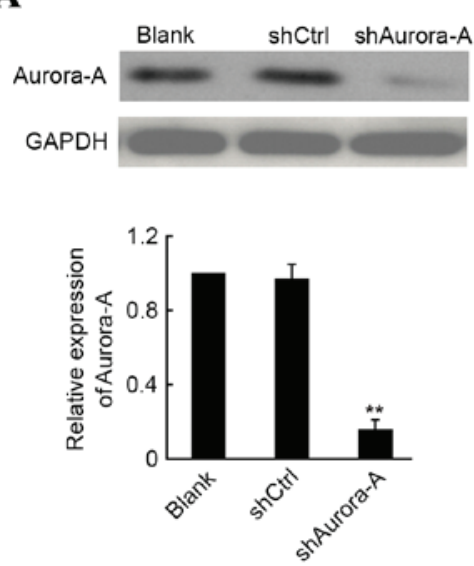

C U251

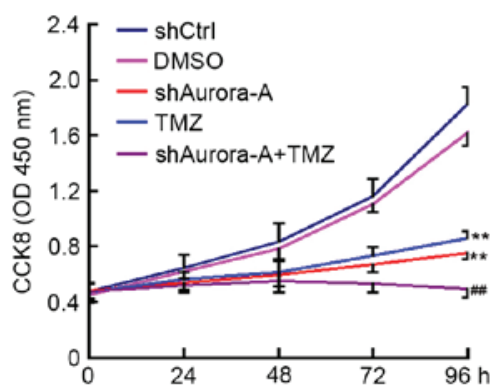

B
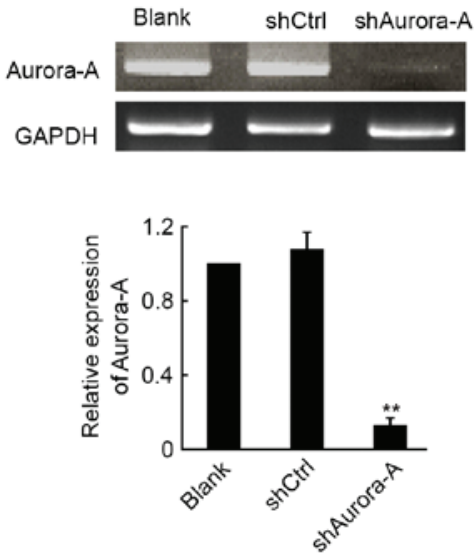

D

U87-MG

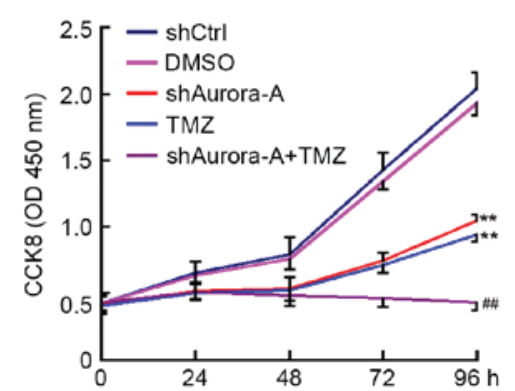

Figure 1. Knockdown of Aurora-A sensitizes glioblastoma cells to TMZ in vitro. U251 cells were transfected with plasmid-based shAurora-A, shCtrl or medium alone as control for $48 \mathrm{~h}$. Aurora-A expression was detected by (A) western blotting or (B) reverse transcription-polymerase chain reaction analysis. GAPDH expression was monitored as the control. The ratio of Aurora-A/GAPDH was calculated using densitometry. The experiments were performed in triplicate and the mean ratio is shown. The transfected (C) U251 and (D) U87-MG cells were plated for CCK8 assay and treated with or without TMZ. DMSO

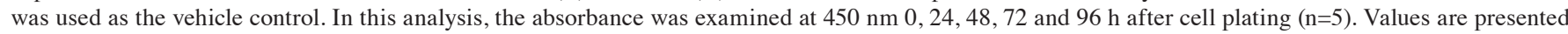
as the mean \pm standard deviation. The experiment was performed in triplicate. ${ }^{* * *} \mathrm{P}<0.01$ compared with shCtrl group; ${ }^{\# /} \mathrm{P}<0.01$ compared with TMZ group. sh, short hairpin; Ctrl, control; GAPDH; glyceraldehyde 3-phosphate dehydrogenase; CCK8, cell counting Kit-8; TMZ, temozolomide; DMSO, dimethyl sulfoxide; OD, optical density.

using a DeadEnd ${ }^{\mathrm{TM}}$ Fluorometric TUNEL System (Promega Corporation, Madison, WI, USA). Cell nuclei with dark green fluorescent staining were defined as TUNEL-positive. TUNEL-positive nuclei were monitored by fluorescence microscopy. To quantify TUNEL-positive cells, the number of green fluorescence-positive cells was counted in random fields at magnification, $\mathrm{x} 200$.

Statistical analysis. Data are expressed as the mean \pm standard deviation. Statistical analysis was performed by Student's t-test for comparing two groups and by analysis of variance for multiple group comparisons with one-way analysis of variance. $\mathrm{P}<0.05$ was considered to indicate a statistically significant difference. SPSS version 19 (IBM SPSS, Armonk, NY, USA) was used for all statistical analyses.

\section{Results}

Knockdown of Aurora-A sensitizes glioblastoma cells to TMZ in vitro. The shAurora-A and shCtrl plasmids were transfected into U251 GBM cancer cells. A total of $48 \mathrm{~h}$ later, the cells were harvested and the expression level of Aurora-A was analyzed by western blotting and RT-PCR. As shown in Fig. 1A and $\mathrm{B}$, suppression of Aurora-A expression was observed in U251 cells transfected with shAurora-A plasmid at the protein and mRNA level when compared with the control group. Subsequently, the effect of shAurora-A combined with TMZ on U251 and U87-MG cell proliferation was examined by CCK8 assay. The absorbance was determined at $0,24,48,72$ and $96 \mathrm{~h}$ following U251 and U87-MG cell transfection and treatment with TMZ. As shown in Fig. 1C and D, knockdown of Aurora-A significantly inhibits U251 $(\mathrm{P}=0.0062)$ and U87-MG $(\mathrm{P}=0.0072)$ cancer cell growth. Furthermore, TMZ treatment alone may inhibit U251 and U87-MG cancer cell growth. In addition, the $\mathrm{U} 251(\mathrm{P}=0.0042)$ and $\mathrm{U} 87-\mathrm{MG}(\mathrm{P}=0.0059)$ cells that were transfected with shAurora-A and treated with TMZ demonstrated significantly increased inhibition of proliferation compared with the group treated with TMZ alone. The above results indicate that knockdown of Aurora-A sensitizes GBM cells to TMZ in vitro.

Knockdown of Aurora-A increases the effects of TMZ treatment on GBM cell apoptosis and colony formation. The quantitative assessment of sub-G1 cells by FCM was used to estimate the number of apoptotic cells. As shown in Fig. 2A and B, an increase in the sub-G1 phase population was observed by flow cytometric analysis following knockdown of Aurora-A or treatment with TMZ in U251 cell lines. 


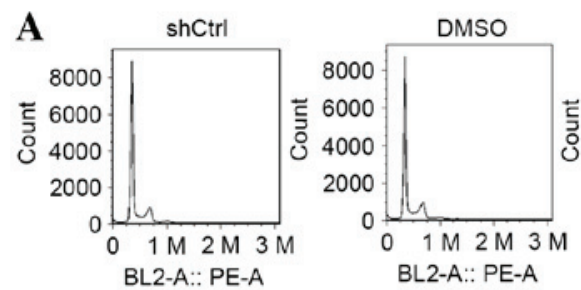

B

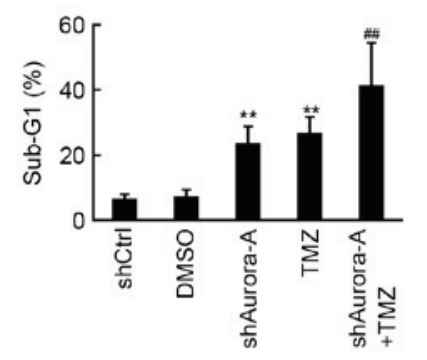

D
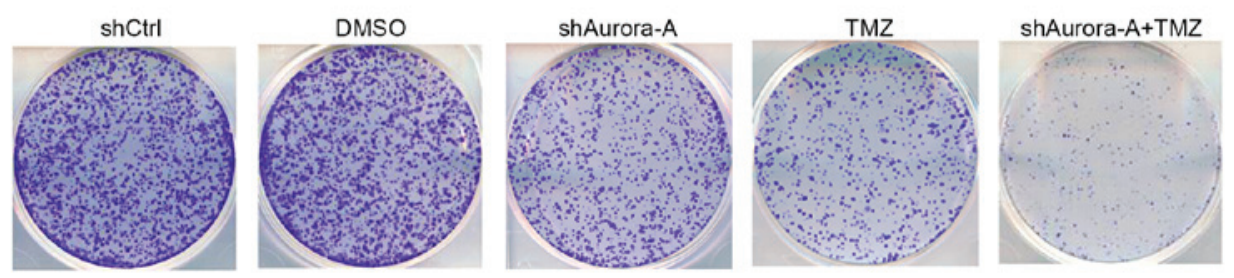

E

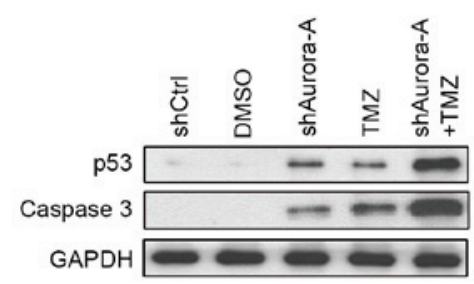

Figure 2. Effects of shAurora-A on U251 apoptosis and colony formation. (A) Cells were collected 48 h following transfection and treatment with or without TMZ, and subsequently assayed for their DNA content by flow cytometry. These experiments were performed in triplicate. (B) The mean percentages of sub-G1 population are shown. Values are presented as the mean $\pm \mathrm{SD}$. ${ }^{* *} \mathrm{P}<0.01$ compared with shCtrl group; ${ }^{\# \#} \mathrm{P}<0.01$ compared with $\mathrm{TMZ}$ group. $(\mathrm{C}) \mathrm{The}$ colony formation number was calculated from colony formation assays (as presented in D). Values are presented as the mean $\pm \mathrm{SD}$. ${ }^{* *} \mathrm{P}<0.01$ compared with shCtrl group; ${ }^{\#} \mathrm{P}<0.01$ compared with TMZ group. (D) Following transfection cells were plated in 6-well plates and treated with or without TMZ. A total of 7 days later, cells were stained with $0.05 \%$ crystal violet. Colony formation assays for each group were performed. These experiments were performed in triplicate. (E) Western blot analysis was performed to measure expression of the apoptosis-associated proteins p53 and caspase-3 in each group. sh, short hairpin; TMZ, temozolomide; SD, standard deviation; DMSO, dimethyl sulfoxide; Ctrl, control.

Furthermore, U251 cells transfected with shAurora-A exhibited significantly increased apoptosis $(\mathrm{P}=0.0007)$ compared with the TMZ-treated group. As shown in Fig. $2 \mathrm{C}$ and $\mathrm{D}$, the inhibition of U251 cell colony formation in the shAurora-A and TMZ-treated group was measured, and compared with the shCtrl and DMSO groups, respectively. Additionally, knockdown of Aurora-A increased the inhibitive effect of TMZ treatment on U251 colony formation, compared with the group treated with TMZ alone. The results of western blot analysis shown in Fig. 2E indicated that shAurora-A $(\mathrm{P}=0.0038)$ or TMZ ( $\mathrm{P}=0.0009)$ treatment alone significantly induced the apoptosis-associated protein p53, as well as caspase 3 expression. Furthermore, increased p53 and caspase 3 expression was observed in the shAurora-A+TMZ group. The above results indicate that knockdown of Aurora-A increases the effects of TMZ on GBM cell apoptosis and colony formation through induced p53 and caspase 3 expression.
Knockdown of Aurora-A increases the effects of TMZ treatment on GBM cell invasion and angiogenesis. Invasion and tube formation assays were performed to determine whether there is a synergistic effect in the group knockdown of Aurora-A in U251 cells combined with TMZ treatment. As shown in Fig. 3A and B, knockdown of Aurora-A $(\mathrm{P}=0.0029)$ and TMZ treatment $(\mathrm{P}=0.023)$ alone significantly inhibited U251 invasion, compared with the shCtrl or DMSO groups, respectively. Furthermore, fewer invasive cells were observed in the shAurora-A combined with TMZ treatment group, when compared with the TMZ only group. Subsequently, western blotting results indicated that MMP-2 and MMP-9 levels were inhibited following shAurora-A transfection (Fig. 3C). The conditional medium from each group was used to perform the HUVEC tube formation assay. The results of the present study demonstrated that the conditional medium from the shAurora-A $(\mathrm{P}=0.0026)$ or $\mathrm{TMZ}(\mathrm{P}=0.017)$ treated groups 
A

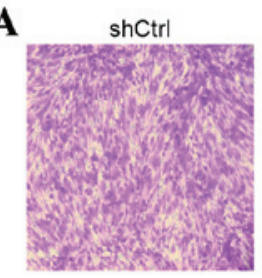

B

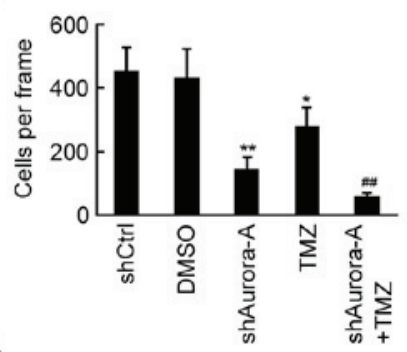

D

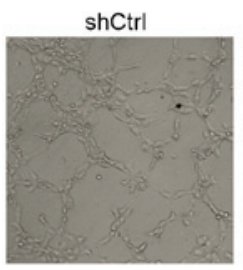

$\mathbf{E}$

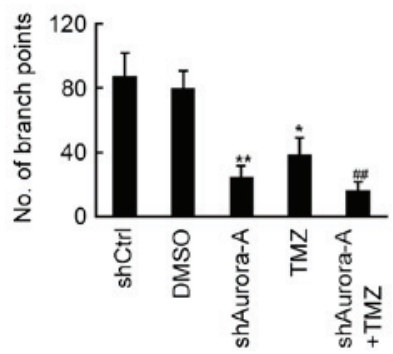

shAurora-A
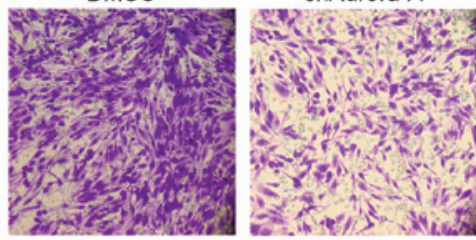

C

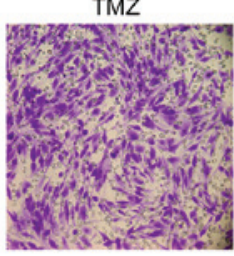

shAurora-A+TMZ
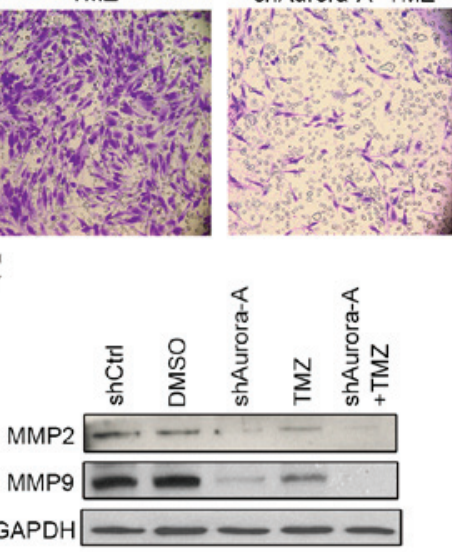

TMZ
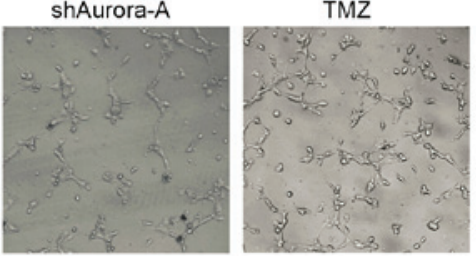

shAurora-A+TMZ

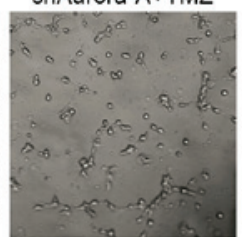

F

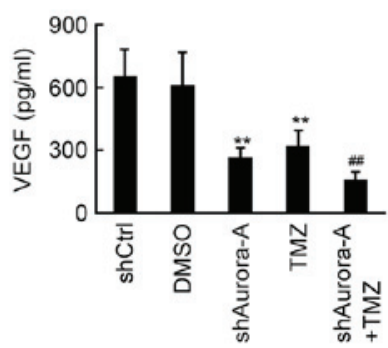

Figure 3. Effects of shAurora-A on U251 invasion and tube formation. (A) Cell invasion in U251 cells was evaluated using Transwell chambers. These experiments were performed in triplicate (magnification, x200). (B) The average number of invasive cells is shown. Values are presented as the mean \pm SD. ${ }^{* *} \mathrm{P}<0.01$ compared with shCtrl group; ${ }^{*} \mathrm{P}<0.05$ compared with shCtrl group; ${ }^{\# \#} \mathrm{P}<0.01$ compared with TMZ group. (C) Western blot analysis was performed to measure the expression of the invasion-associated proteins MMP-2 and MMP-9 in each group. (D) Conditional medium from each group was used for tube formation assays in human umbilical vein endothelial cells. These experiments were performed in triplicate (magnification, $\mathrm{x} 200$ ). (E) The tubular structure in each group was quantified by manual counting. The mean number of branch points is shown. Values are presented as the mean $\pm \mathrm{SD}$. ${ }^{* *} \mathrm{P}<0.01 \mathrm{compared}$ with shCtrl group; ${ }^{*} \mathrm{P}<0.05$ compared with shCtrl group; ${ }^{\#} \mathrm{P}<0.01$ compared with TMZ group. (F) VEGF expression from the conditional medium of each group was determined via enzyme-linked immunosorbent assay. These experiments were performed in triplicate. The average concentrations of VEGF are shown. Values are presented as the mean $\pm \mathrm{SD}$. ${ }^{* *} \mathrm{P}<0.01$ compared with shCtrl group; ${ }^{\# \#} \mathrm{P}<0.01$ compared with TMZ group. sh, short hairpin; SD, standard deviation; TMZ, temozolomide; MMP, matrix metallopeptidase; Ctrl, control; DMSO, dimethyl sulfoxide; GAPDH, glyceraldehyde 3-phosphate dehydrogenase; VEGF, vascular endothelial growth factor.

significantly inhibited HUVEC tube formation, compared with the shCtrl or DMSO groups, respectively (Fig. 3D and E). Furthermore, knockdown of Aurora-A increased the inhibition of tube formation in HUVECs, compared with the TMZ-treated group (Fig. 3D and E). Subsequently, VEGF expression for each group was determined by ELIZA assay and decreased VEGF was observed in the shAurora-A group compared with the shCtrl group. A further decrease in VEGF expression was observed in the shAurora-A+TMZ-treated group when compared with the TMZ-treated alone group (Fig. 3D and E). The above results demonstrate that knockdown of Aurora-A increases the effects of TMZ treatment on GBM cell invasion and angiogenesis through the inhibition of MMP-2, MMP-9 and VEGF expression.
Knockdown of Aurora-A sensitizes GBM cells to TMZ treatment in mice. The present study determined the effects of silencing Aurora-A on tumor growth and in mice through plasmid-based shRNA. A U251 GBM subcutaneous mouse model was used. A plasmid/liposome complex was systemically injected through the mouse tail vein, combined with or without TMZ treatment. As shown in Fig. 4A, injection of the shAurora-A/liposome complex significantly inhibited U251 subcutaneous growth, compared with the shCtrl/liposome complex group $(n=5$, tumor volume: shAurora-A/liposome group, 1,063.4 $\pm 79.3 \mathrm{~mm}^{3}$ vs. shCtrl/liposome group, 2,103.2 $\pm 216.4 \mathrm{~mm}^{3} ; \mathrm{P}=0.0051$ ). Furthermore, TMZ treatment also caused inhibition of U251 tumor growth compared with the DMSO group $(n=5$, tumor volume: TMZ group, $894.6 \pm 68.9 \mathrm{~mm}^{3}$ vs. DMSO group, 
$\mathbf{A}$
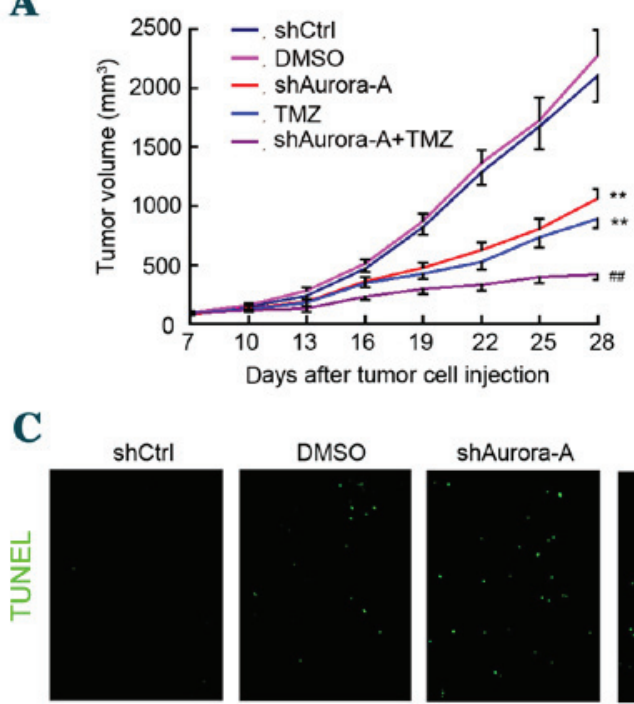

$\mathbf{E}$

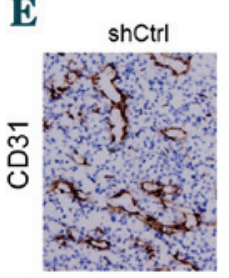

DMSO

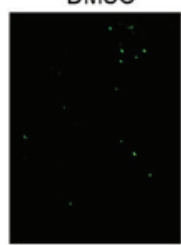

shAurora-A
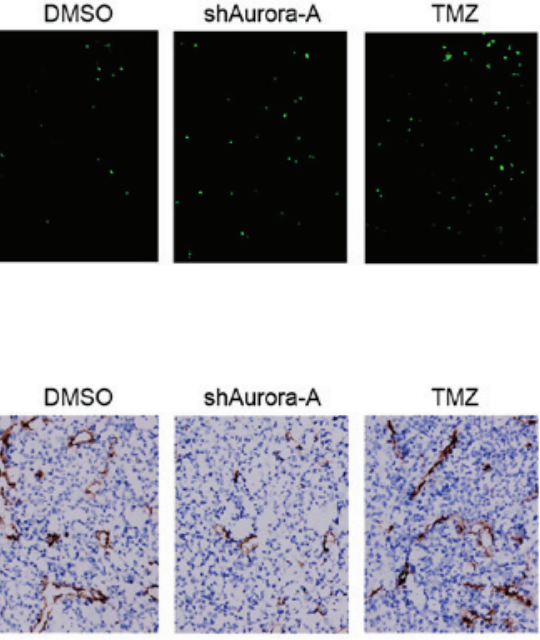

TMZ

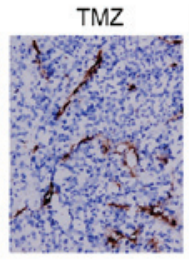

B

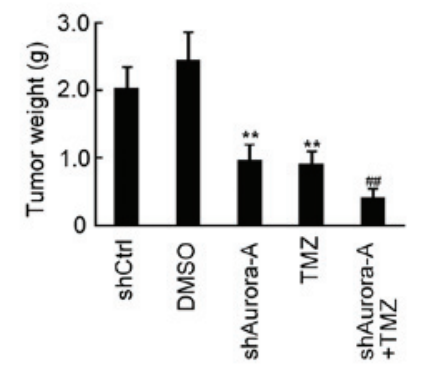

D
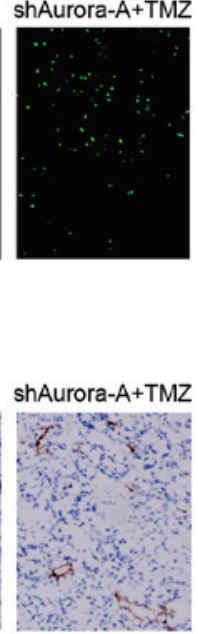

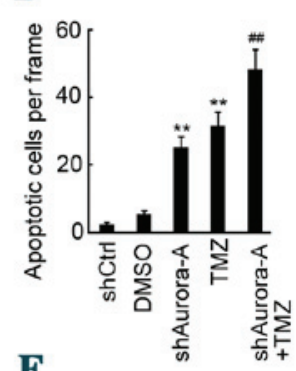

F

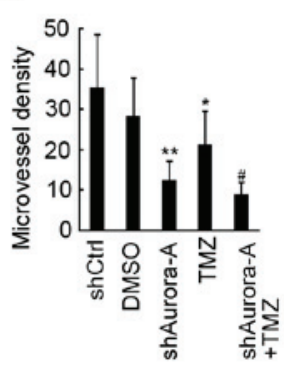

Figure 4. Knockdown of Aurora-A sensitizes glioblastoma cells to TMZ in mice. (A) Female nude mice at 6-8 weeks of age were implanted subcutaneously with U251 cells. A total of 7 days after tumor cells were implanted, the mice were assigned randomly to five groups and treated with an shAurora-A/liposome or shCtrl/liposome complex, combined with or without TMZ. Tumor volume was recorded during the treatment and growth curves were constructed. Values are presented as the mean $\pm \mathrm{SD}(\mathrm{n}=5) .{ }^{* *} \mathrm{P}<0.01$ compared with shCtrl group; ${ }^{\# \#} \mathrm{P}<0.01$ compared with TMZ group. (B) A total of 3 days after the final treatment, mice were sacrificed and subcutaneous tumors were weighed. Values are presented as the mean $\pm \mathrm{SD}(\mathrm{n}=5) .{ }^{* *} \mathrm{P}<0.01$ compared with shCtrl group; ${ }^{\# \#} \mathrm{P}<0.01$ compared with TMZ group. (C and D) The TUNEL assay was performed to detect apoptotic cells in primary U251 xenografts (magnification, $\mathrm{x} 100$ ). The apoptotic index was calculated as a ratio of the apoptotic cell number to the total cell number in each field. Values are presented as the mean \pm SD ( $\mathrm{n}=5$ ). ${ }^{* *} \mathrm{P}<0.01$ compared with shCtrl group; ${ }^{\# \#} \mathrm{P}<0.01$ compared with TMZ group. (E) Cluster of differentiation 31 staining was performed to detect angiogenesis in primary U251 xenografts (magnification, $\mathrm{x} 200)$. (F) The microvessel density was calculated. Values are presented as the mean $\pm \mathrm{SD}(\mathrm{n}=5)$. ${ }^{* *} \mathrm{P}<0.01 \mathrm{compared}$ with shCtrl group; ${ }^{*} \mathrm{P}<0.05$ compared with shCtrl group; ${ }^{\# \#} \mathrm{P}<0.01$ compared with TMZ group. TMZ, temozolomide; sh, short hairpin; $\mathrm{SD}$, standard deviation; DMSO, dimethyl sulfoxide; Ctrl, control.

2,275.4 $\left.\pm 221.4 \mathrm{~mm}^{3} ; \mathrm{P}=0.0036\right)$. Notably the shAurora-A/liposome complex combined with TMZ treatment significantly inhibited U251 tumor growth compared with the TMZ only group ( $\mathrm{n}=5$, tumor volume: Combined group, $422.6 \pm 48.3 \mathrm{~mm}^{3}$ vs. TMZ group, $894.6 \pm 68.9 \mathrm{~mm}^{3}$; $\left.\mathrm{P}=0.0084\right)$. Furthermore, U251 tumor weight was also inhibited by combined shAurora-A/liposome complex and TMZ treatment, compared with shCtrl/liposome and DMSO groups, respectively (Fig. 4B). The combined treatment group also displayed markedly decreased tumor weight, when compared with the TMZ only group $(n=5$, tumor weight: Combined group, $0.41 \pm 0.13 \mathrm{~g}$ vs. TMZ group $0.91 \pm 0.18$ g; $\mathrm{P}=0.0067$; Fig. 4B).

To detect apoptotic cells in tumor tissue, the TUNEL assay was performed. As shown in Fig. 4C and D, an increased number of apoptotic cells was observed in the shAurora-A/liposome complex+TMZ group, compared with the shCtrl/liposome complex or DMSO groups, respectively $(n=5 ; P=0.0052)$. Furthermore, there was an increased number of apoptotic cells in the shAurora-A/liposome complex+TMZ group, compared with the TMZ only group $(n=5 ; P=0.0037)$. CD31 staining indicated that fewer microvessels were observed in the shAurora-A/liposome complex+TMZ group, compared with the shCtrl/liposome complex or DMSO groups, respectively $(n=5 ; \mathrm{P}=0.0028$; Fig. 4E and F). Furthermore, there were fewer microvessels in the shAurora-A/liposome complex+TMZ group, compared with the TMZ only group $(n=5 ; P=0.0042$; Fig. 4E and $F)$. The above results indicate that knockdown of Aurora-A sensitizes GBM cells to TMZ in mice by inducing apoptosis and inhibiting angiogenesis.

\section{Discussion}

The present study demonstrates that the therapeutic knockdown of Aurora-A through plasmid-based shAurora-A sensitizes GBM cells to TMZ chemotherapy in vitro and in vivo. The 
plasmid encoding shRNA targeting Aurora-A was proven to knockdown Aurora-A expression at the mRNA and protein level in U251 cells. Furthermore, CCK8 assays, flow cytometric analysis, colony formation assays, invasion assays and tube formation assays were used to demonstrate that knockdown of Aurora-A sensitizes GBM cells to TMZ in vitro. Notably, TMZ combined with knockdown of Aurora-A through plasmid based shAurora-A/liposome complex injection, significantly inhibited U251 subcutaneous tumor growth compared with TMZ treatment alone.

Aurora-A is widely expressed in several types of cancer and has been observed to be inversely correlated with patient prognosis $(14,15)$. Previous studies indicated that Aurora-A is essential for the tumorigenic capacity and chemoresistance of colorectal cancer stem cells (26). Thus, Aurora-A may be an effective target for gene therapy and chemotherapy. A study by Van Brocklyn et al (14) indicated that knockdown of Aurora-A in GBM by alisertib (MLN8237), a highly selective Aurora-A kinase inhibitor, extended the median survival time of mice bearing intracranial human GBM neurosphere tumor xenografts. However, whether knockdown of Aurora-A sensitizes GBM cells to TMZ treatment remains to be elucidated. In the present study, plasmid-based shRNA targeting Aurora-A was used to inhibit its expression in GBM cells. The results of the present study indicate that shAurora-A significantly inhibits the expression of Aurora-A at the mRNA and protein level. Knockdown of Aurora-A may significantly inhibit glioblastoma cell proliferation, colony formation, invasion, angiogenesis and apoptosis induction in vitro and in vivo. Further results demonstrate that inhibition of Aurora-A in U251 cells induces expression of the pro-apoptotic proteins p53 and caspase 3. Furthermore, MMP-2, MMP-9 and VEGF expression were decreased by shAurora-A.

TMZ is a widely used chemotherapeutic agent in the treatment of GBM (4). Despite the clinical occurrence of TMZ resistance (27), the dose of TMZ used significantly affects the patient quality of life $(8,9)$. Thus, in recent years studies have focused on developing novel treatment options to sensitize GBM cells to TMZ. Several inhibitors, including KML001 and nelfinavir were proven to sensitize GBM cells to TMZ treatment $(28,29)$. In the present study, TMZ treatment alone was proven to significantly inhibit glioblastoma cell proliferation, colony formation, invasion, angiogenesis, and apoptosis induction in vitro and in vivo. These observations are consistent with the results of previous studies $(30,31)$. Notably, knockdown of Aurora-A in GBM cells greatly enhances TMZ sensitivity in vitro and in vivo, compared with TMZ treatment alone. This is due to the altered expression of Aurora-A regulated proteins, including p53, caspase 3, MMP-2, MMP-9 and VEGF.

The results of the present study demonstrate that knockdown of Aurora-A in GBM cells greatly enhances TMZ sensitivity in vitro and in vivo. This may present a novel treatment option for decreasing TMZ toxicity and improving patient quality of life.

\section{Acknowledgements}

The present work was supported by the National Science Foundation of China (grant nos. 81330,016 and 31171020 to
Dr Dezhi Mu; grant nos. 81172174 and 81270724 to Dr Yi Qu), the Major State Basic Research Development Program (grant no. 2013CB967404), grants from the Ministry of Education of China (grant nos. 313037 and 20110181130002 ), the grant from the State Commission of Science Technology of China (grant nos. 2012BAI04B04 and 2012BAI04B04), the grant from the Science and Technology Bureau of Sichuan province (grant no. 2011JTD0005), the National Science Foundation of China, (grant no. 81501301 to Mr. Jing Gan) and the grant of the clinical discipline program (neonatology) from the Ministry of Health of China (grant no. 1311200003303).

\section{References}

1. Sathornsumetee S and Rich JN: New treatment strategies for malignant gliomas. Expert Rev Anticancer Ther 6: 1087-1104, 2006.

2. Mrugala MM and Chamberlain MC: Mechanisms of disease: Temozolomide and glioblastoma-look to the future. Nat Clin Pract Oncol 5: 476-486, 2008.

3. Friedman HS, Kerby T and Calvert H: Temozolomide and treatment of malignant glioma. Clin Cancer Res 6: 2585-2597, 2000.

4. Osoba D, Brada M, Yung WK and Prados M: Health-related quality of life in patients treated with temozolomide versus procarbazine for recurrent glioblastoma multiforme. J Clin Oncol 18: 1481-1491, 2000.

5. Margison GP, Santibáñez Koref MF and Povey AC: Mechanisms of carcinogenicity/chemotherapy by O6-methylguanine. Mutagenesis 17: 483-487, 2002.

6. Hirose Y, Berger MS and Pieper RO: p53 effects both the duration of $\mathrm{G} 2 / \mathrm{M}$ arrest and the fate of temozolomide-treated human glioblastoma cells. Cancer Res 61: 1957-1963, 2001.

7. Quiros S, Roos WP and Kaina B: Processing of O6-methylguanine into DNA double-strand breaks requires two rounds of replication whereas apoptosis is also induced in subsequent cell cycles. Cell Cycle 9: 168-178, 2010.

8. Ahluwalia MS, Xie H, Dahiya S, Hashemi-Sadraei N, Schiff D, Fisher PG, Chamberlain MC, Pannullo S, Newton HB, Brewer C, et al: Efficacy and patient-reported outcomes with dose-intense temozolomide in patients with newly diagnosed pure and mixed anaplastic oligodendroglioma: A phase II multicenter study. J Neurooncol 122: 111-119, 2015.

9. Taphoorn MJ, Henriksson R, Bottomley A, Cloughesy T, Wick W, Mason WP, Saran F, Nishikawa R, Hilton M, Theodore-Oklota C, et al: Health-Related quality of life in a randomized phase III study of bevacizumab, temozolomide, and radiotherapy in newly diagnosed glioblastoma. J Clin Oncol 33: 2166-2175, 2015.

10. Marumoto T, Honda S, Hara T, Nitta M, Hirota T, Kohmura E and Saya H: Aurora-A kinase maintains the fidelity of early and late mitotic events in HeLa cells. J Biol Chem 278: 51786-51795, 2003.

11. Seki A, Coppinger JA, Jang CY, Yates JR and Fang G: Bora and the kinase Aurora a cooperatively activate the kinase Plk1 and control mitotic entry. Science 320: 1655-1658, 2008.

12. Zhou H, Kuang J, Zhong L, Kuo WL, Gray JW, Sahin A, Brinkley BR and Sen S: Tumour amplified kinase STK15/BTAK induces centrosome amplification, aneuploidy and transformation. Nature Genet 20: 189-193, 1998.

13. Sen S, Zhou $\mathrm{H}$ and White RA: A putative serine/threonine kinase encoding gene BTAK on chromosome $20 \mathrm{q} 13$ is amplified and overexpressed in human breast cancer cell lines. Oncogene 14: 2195-2200, 1997.

14. Van Brocklyn JR, Wojton J, Meisen WH, Kellough DA, Ecsedy JA, Kaur B and Lehman NL: Aurora-A inhibition offers a novel therapy effective against intracranial glioblastoma. Cancer Res 74: 5364-5370, 2014.

15. Yang H, Ou CC, Feldman RI, Nicosia SV, Kruk PA and Cheng JQ: Aurora-A kinase regulates telomerase activity through c-Myc in human ovarian and breast epithelial cells. Cancer Res 64: 463-467, 2004.

16. Katayama H, Sasai K, Kawai H, Yuan ZM, Bondaruk J, Suzuki F, Fujii S, Arlinghaus RB, Czerniak BA and Sen S: Phosphorylation by aurora kinase A induces Mdm2-mediated destabilization and inhibition of p53. Nat Genet 36: 55-62, 2004.

17. Qin L, Tong T, Song Y, Xue L, Fan F and Zhan Q: Aurora-A interacts with Cyclin B1 and enhances its stability. Cancer Lett 275: 77-85, 2009. 
18. Sasayama T, Marumoto T, Kunitoku N, Zhang D, Tamaki N, Kohmura E, Saya H and Hirota T: Over-expression of Aurora-A targets cytoplasmic polyadenylation element binding protein and promotes mRNA polyadenylation of Cdk1 and cyclin B1. Genes Cells 10: 627-638, 2005.

19. Yang S, He S, Zhou X, Liu M, Zhu H, Wang Y, Zhang W, Yan S, Quan L, Bai J and Xu N: Suppression of Aurora-A oncogenic potential by c-Myc downregulation. Exp Mol Med 42: 759-767, 2010.

20. Carol H, Boehm I, Reynolds CP, Kang MH, Maris JM, Morton CL, Gorlick R, Kolb EA, Keir ST, Wu J, et al: Efficacy and pharmacokinetic/pharmacodynamic evaluation of the Aurora kinase A inhibitor MLN8237 against preclinical models of pediatric cancer. Cancer Chemother Pharmacol 68: 1291-1304, 2011.

21. Huck JJ, Zhang M, McDonald A, Bowman D, Hoar KM, Stringer B, Ecsedy J, Manfredi MG and Hyer ML: MLN8054, an inhibitor of Aurora A kinase, induces senescence in human tumor cells both in vitro and in vivo. Mol Cancer Res 8: 373-384, 2010.

22. Hata T, Furukawa T, Sunamura M, Egawa S, Motoi F, Ohmura N, Marumoto T, Saya $\mathrm{H}$ and Horii A: RNA interference targeting aurora kinase a suppresses tumor growth and enhances the taxane chemosensitivity in human pancreatic cancer cells. Cancer Res 65 : 2899-2905, 2005

23. Dai L, Cui X, Zhang X, Cheng L, Liu Y, Yang Y, Fan P, Wang Q, Lin Y,Zhang J, et al: SARI inhibits angiogenesis and tumour growth of human colon cancer through directly targeting ceruloplasmin. Nat Commun 7: 1996, 2016.

24. Dai L, Cheng L, Zhang X, Jiang Q, Zhang S, Wang S, Li Y, Chen X, Du T, Yang Y, et al: Plasmid-based STAT3-siRNA efficiently inhibits breast tumor growth and metastasis in mice. Neoplasma 58: 538-547, 2011.

25. Wang W, Dai LX, Zhang S, Yang Y, Yan N, Fan P, Dai L, Tian HW, Cheng L, Zhang XM, et al: Regulation of epidermal growth factor receptor signaling by plasmid-based microRNA-7 inhibits human malignant gliomas growth and metastasis in vivo. Neoplasma 60: 274-283, 2013.
26. Cammareri P, Scopelliti A, Todaro M, Eterno V, Francescangeli F, Moyer MP, Agrusa A, Dieli F, Zeuner A and Stassi G: Aurora-a is essential for the tumorigenic capacity and chemoresistance of colorectal cancer stem cells. Cancer Res 70: 4655-4665, 2010.

27. Hegi ME, Liu L, Herman JG, Stupp R, Wick W, Weller M, Mehta MP and Gilbert MR: Correlation of O6-methylguanine methyltransferase (MGMT) promoter methylation with clinical outcomes in glioblastoma and clinical strategies to modulate MGMT activity. J Clin Oncol 26: 4189-4199, 2008.

28. Jiang Z, Pore N, Cerniglia GJ, Mick R, Georgescu MM, Bernhard EJ, Hahn SM, Gupta AK and Maity A: Phosphatase and tensin homologue deficiency in glioblastoma confers resistance to radiation and temozolomide that is reversed by the protease inhibitor nelfinavir. Cancer Res 67: 4467-4473, 2007.

29. Woo SR, Ham Y, Kang W, Yang H, Kim S, Jin J, Joo KM and Nam DH: KML001, a telomere-targeting drug, sensitizes glioblastoma cells to temozolomide chemotherapy and radiotherapy through DNA damage and apoptosis. Biomed Res Int 2014: 747415, 2014

30. Goellner EM, Grimme B, Brown AR, Lin YC, Wang XH, Sugrue KF, Mitchell L, Trivedi RN, Tang JB and Sobol RW: Overcoming temozolomide resistance in glioblastoma via dual inhibition of NAD+ biosynthesis and base excision repair. Cancer Res 71: 2308-2317, 2011.

31. Park I, Mukherjee J, Ito M, Chaumeil MM, Jalbert LE, Gaensler K, Ronen SM, Nelson SJ and Pieper RO: Changes in pyruvate metabolism detected by magnetic resonance imaging are linked to DNA damage and serve as a sensor of temozolomide response in glioblastoma cells. Cancer Res 74: 7115-7124, 2014. 